\title{
The Hierarchical Ad Hoc Networks Anonymous Routing Scheme
}

\author{
Yuan Xiaopeng, Wu Jianjun, Guo Xian, Feng Tao, Li Xinghua, and Wang Jing
}

\begin{abstract}
Anonymous routing protocol is designed for avoiding from being leaked by nodes during communication in mobile ad hoc networks and insuring the communication route can't be discovered. Whereas most proposed anonymous secure routing protocols known in Ad Hoc networks can't meet anonymity sufficient or apply to hierarchical Ad Hoc networks. In addition, the protocols almost didn't have formal proof. Our mechanism based on ring signature, looking for honest neighbors. The source node set "trap-door" by using the public key of destination. Adopt the on-demand routing protocol to complete this program. This new protocols meet the hierarchical Ad Hoc networks by joining the network identifier. Finally, we adopt the formal proof tool UC(universally composable model) to prove the safety and anonymity of the new protocols.
\end{abstract}

Index Terms-Ad hoc, ring signature, UC, anonymous routing.

\section{INTRODUCTION}

Anonymous communication was first proposed by Chaum [1]. Depending on the object of protection, anonymous form of protection can be divided into three types: sender anonymity, recipient anonymity and relationship anonymity. Researchers made improvements in the classic on-demand routing protocols AODV and DSR in order to meet anonymous communication. Such as ANODR ASR, MASK [2]. However most of these protocols only considered relationship anonymity. Intermediate nodes communication will bring malicious nods, which may lead to information leaking that can't meet anonymity sufficient. Lin et al. [3] proposed an anonymous authentication routing protocol which is based on ring signature. it integrates a suit of interoperative authenticated key exchange mechanisms into the routing algorithm design, which can insure the intermidate nods are honst nodes. Whereas the proposed ring signature can't unforgeable against chosen-subring attacks [4]. In this

Manuscript received June 13, 2014; revised September 2, 2014. This work was supported in by The National Natural Science Foundation of China under Grant 61462060 and 61461027 . This work also supported in by Science and Technology Program of Gansu Province under Grant 1308RJZA277 and 145RJZA078.

Yuan xiaopeng is with High-level School Admissions Office of Gansu Province, Lanzhou, China.

Wu Jianjun, Guo Xian, and Wang jing are with School of Computer and Communication Lanzhou University of Technology, Lanzhou, China (e-mail: wangjing@lut.cn).

Feng Tao is with School of Computer and Communication Lanzhou University of Technology, Lanzhou, he is also with the Ministry of Education Key Laboratory of Computer Networks and Information Security Xidian University, Xi'an, China.

Li Xinghua is with the Ministry of Education Key Laboratory of Computer Networks and Information Security Xidian University, Xi'an, China. paper will introduce a new anonymous routing scheme which has three advantages: (1) it can provides not only anonymity to the route from source to destination, but sender anonymity. (2) it is suitable for hierarchical ad hoc networks. (3) it achieves universally composable security.

\section{PRELIMINARIES}

Ring Signature. Bender et al. [4] gave a strict definition of the ring signature. Kazuki et al. [5] gave an ideal function of UC security according to the anonymous program to achieve anonymity and unforgeability. They also proved the Bender's protocols meet the UC security.

Gen: Generate signing key pair $\left(p k_{s}, s k_{s}\right) \leftarrow \operatorname{Gen}^{\prime}\left(1^{k}\right)$, Generate encryption key pair $\left(p k_{E}, s k_{E}\right) \leftarrow \operatorname{EGen}^{\prime}\left(1^{k}\right)$ and erase $s k_{E}$, Choose an initial ZAP (2-round, public-coin, witness indistinguishable proof system) message $r \leftarrow\{0,1\}^{l(k)}$, Output the public key $P K:=\left(p k_{S}, p k_{E}, r\right)$, and the secret key $S K:=s k_{S}$.

Sign: Parse each $P K_{i}$ as $\left(p k_{S . i}, p k_{E, i}, r_{i}\right)$, and parse $S K_{i^{*}}$ as $S K_{S, i^{*}}$. Set $R_{E}:=\left\{p k_{E, 1}, 1, \cdots p k_{E, n}\right\}$ and $R_{S}=\left\{p k_{S, 1}, 1, \cdots p k_{S, n}\right\}$. Set $M^{*}:=M\left|P K_{1}\right| \cdots \mid P K_{n}$, where "|" denotes concatenation. Compute the signature $\sigma_{i^{*}}^{\prime} \leftarrow \operatorname{Sign}_{s k_{S, i^{*}}}^{\prime}\left(M^{*}\right)$. Choose a random coins $\omega_{0}, \omega_{1}$ and compute $C_{0}^{*}:=E n c_{R_{E}}^{*}\left(\sigma_{i^{*}}^{\prime} ; \omega_{0}\right)$ and $C_{1}^{*}: E n c_{R_{E}}^{*}\left(0^{k} ; \omega_{0}\right)$. For $j \in\{0,1\}$, let $x_{j}$ denote the statement: " $\left(R_{S}, M^{*}, R_{E}, C_{j}^{*}\right) \in L$ ”, and let $x:=x_{0} \vee x_{1}$. compute the proof $\pi \leftarrow P_{r l}\left(x,\left(p k_{s, i^{*}}, \sigma_{i^{*}}^{\prime}, \omega_{0}\right)\right)$. The signature is $\sigma:=\left(C_{0}^{*}, C_{1}^{*}, \pi\right)$.

Verify: Parse $\sigma$ as $\left(C_{0}^{*}, C_{1}^{*}, \pi\right)$. Parse each $P K_{i}$ as $\left(p k_{S . i}, p k_{E, i}, r_{i}\right)$. Set $M^{*}:=M\left|P K_{1}\right| \cdots \mid P K_{n} \quad ; \quad$ set $R_{E}:=\left\{p k_{E, 1}, 1, \cdots p k_{E, n}\right\}$; and set $R_{S}=\left\{p k_{S, 1}, 1, \cdots p k_{S, n}\right\}$. For $j \in\{0,1\}$, let $x_{j}$ denote the statement " $\left(R_{S}, M^{*}, R_{E}, C_{j}^{*}\right) \in L$ ”, and let $x:=x_{0} \vee x_{1}$. Output $v_{r 1}(x, \pi)$.

UC Model. Canetti first proposed a universally composable(UC) framework [6], UC model can be combined to ensure the protocol security. Under UC model, the protocol running in the case with other different protocols, or as a system component of a protocol, still security guarantees agreement.

UC Emulation. A protocol $\pi$ UC-realizes an ideal functionality $F$ if for any real-life adversary $A$, there exists an ideal adversary $S$ such that for any environment $Z$, the 
probability that $Z$ is able to distinguish between an interaction with $A$ and real parties running protocol $\pi$ and an interaction with $S$ and dummy parties accessing $F$ in the ideal process is at most a negligible probability.

Composition Theorem. Let $\rho$ be a protocol that securely realizes the ideal functionality $F$, and let $\pi$ be a protocol in the F-hybrid model. We say that $\pi^{\rho / F}$, with the ideal functionality $F$ which is replaced by $\rho$, UC-realizes $\pi$. In particular, if $\pi$ securely realizes the ideal functionality $G$ in the F-hybrid model, then $\pi^{\rho / F}$ securely realizes $G$ from scratch.

\section{ANONYMOUS ROUTING SCHEME FOR HIERARCHICAL AD HOC NETWORKS}

\section{A. Networks of Background}

Here, the background of what we study is based on the hierarchical ad hoc networks [7], Network nodes are divided according multi-frequency approach. Participating in the network nodes can be divided into the cluster head nodes and ordinary nodes. We shall make the following assumptions:

1) A bidirectional link between two mobile nodes within the transmission range can be established

2) Nodes in the networks have enough computing power and decryption operation perform

3) Each node has a unique identity (ID). the source node can get the destination node's ID(if the destination node in the other subgroup, including the network identity $(N F)$ )

4) Each node maintains two tables: one is a local neighborhood table (Fig. 1), another is local route table(Fig. 2) whose format is as follows:

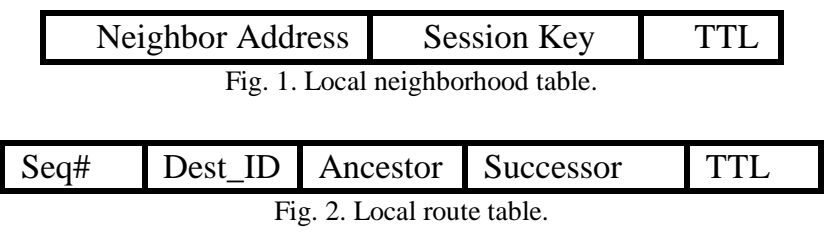

Neighbor Address: neighbor node's address; Session Key: records its session key between itself and the corresponding neighbor, TTL: time to live, if it hits 0 , the table removes. Seq\#: a number represents a unique route, it maintains the route freshness, prevent replay attacks. Dest_ID: the identity of the destination for the source or the identity of the source for the destination. Ancestor: records its upstream node's address. Successor: records its downstream node's.

\section{B. Adversary Model}

Adversary capabilities can generally be divided into passive or active. Attack's goal is to identify the sender and recipient information. Passive attacks monitoring the network traffic data and analysis information. We consider the adversary which controls several nodes of network but does not have full control over the entire network.

Neigborhood Anonymous Authentication Key Exchange Protocol. Cluster head nodes and ordinary nodes do anonymous authentication key exchage protocol based on introduced ring signature. They want to anonymously authenticate each other, where both Alice and Bob know that they are talking to an authentic peer in the ring without knowing the real identity of their peer. They both choose a random number $x \in \mathrm{Z}_{q}^{*}$ and $y \in \mathrm{Z}_{q}^{*}$ and compute $x P, y P$ (where $P$ is the system parameter), when the authentication succeeds, they record address in their trust neighborhood table and possess a new session key at the end of protocol (See Fig. 3).

$$
\begin{aligned}
k_{a b} & =x(y P) \cdots \text { Alice } \\
& =y(x P) \cdots B o b
\end{aligned}
$$

Alice

Bob

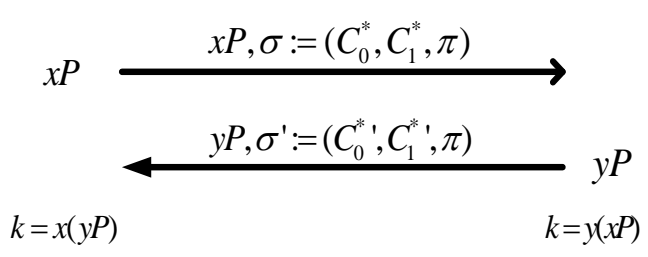

Fig. 3. Key exchange based on the ring signature.

\section{Key Pre-distribution Phase}

We assume an offline security manger (SM) exists for identity check and pre-distribution:

1) $G_{1}$ is an additive group of prime order $q, G_{2}$ is a multiplicative group with the same order as $G_{1}$ and $\hat{e}$ : $G_{1} \times G_{1}, G_{2}$ be the bilinear pairing. Define a secure hash functions $H_{1}:\{0,1\}^{*} \rightarrow G_{1}$;

2) $\mathrm{SM}$ choose random number $s \in Z_{q}^{*}$ as its master key and computes $P_{p u b}=s G$ as its public key;

3) SM calculates for each node an identity-based public/private key pair $\left(P K_{N}, S K_{N}\right)$, where $P K_{N}=H_{1}\left(I D_{N}\right), S K_{N}=s P K_{N}$;

4) Each node is preloaded with the public parameters $<G_{1}, G_{2}, \hat{e}, q, G, P_{p u b}, H_{1}>$ and its private key;

5) SM sends cluster head node its network identifier $(N F)$

\section{Route Discovery Phase}

\section{1) Intra-group anonymous routing request}

Step 1: $S$ generates a unique number seq\#, selects a random number $a \in[1, p-1]$ to compute $g^{a}$ and $H\left(g^{a}\left\|K_{S D}\right\| 0\right)$, where $K_{S D}=\hat{e}\left(H\left(I D_{D}\right), S K_{I D_{S}}\right)$. then $S$ makes $M_{S}=\left[H_{1}\left(I D_{S}\right), I D_{D}, g^{a}, H\left(g^{a}\left\|K_{S D}\right\| 0\right)\right]$, after that ,we use the public key of destination $D$ to encrypt $M_{S}$ as $C_{S}=E_{P K_{D}}\left(M_{S}\right), S$ also sets the number of hops from $S$ to $D$ as HopCount, ARREQ $=<R R E Q$, seq\#, HopCount, Cs $>$ Forward the packet, change its route table as Fig. 4.

Step 2: Upon receiving the ARREQ, intermediate nodes go through the following procedure: Check if it is from one of its trusted neighbor nodes based on the sender's address, if so, it continues. Otherwise it drops. Check if the ARREQ has already been received based on the seq\#, if so, it drops. Otherwise it continues. Check if the node is the destination by decrypting $C_{S}$ with its private key. If the node can decrypt it successfully, the node is receiver; otherwise, the node is not 
the receiver.

If the node is not the intended receiver and (HopCount - -) $\geq 0$, then it forwards the ARREQ to all its neighbors via broadcasting. After execution, intermediate nodes $\left(\mathrm{ID}_{\mathrm{I}}\right)$ change its route table (Fig. 5).

\begin{tabular}{|c|r|c|c|c|}
\hline sequence & Dest_ID & Ancestor & Successor & TTL \\
\hline Seq\# & $\mathrm{ID}_{\mathrm{D}}$ & N/A & $?$ & $\mathrm{~T}_{\mathrm{S}}$ \\
\hline
\end{tabular}

Fig. 4. Source node changes its route table.

\begin{tabular}{|c|c|l|l|c|}
\hline sequence & Dest_ID & Ancestor & Successor & TTL \\
\hline Seq\# & N/A & $\mathrm{I}_{\mathrm{I}-1 \_ \text {addr }}$ & & $\mathrm{T}_{\mathrm{S}}$ \\
\hline \multicolumn{5}{|c}{ Fig. 5. Intermediate node changes its route table. }
\end{tabular}

If the node is the intended receive $\mathrm{D}$, it can correctly recover $M_{S}$ and parse it as $H_{1}\left(I D_{S}\right), I D_{D}, g^{a}$ and $H\left(g^{a}\left\|K_{S D}\right\| 0\right) \quad$ then, it can verify: $H\left(g^{a}\left\|K_{S D}\right\| 0\right)=H\left(g^{a} \| \hat{e}\left(H\left(I D_{S}\right), S K_{I D_{D}} \| 0\right)\right.$ only $S$ and $D$ can compute: $K_{S D}=\hat{e}\left(H\left(I D_{D}\right), S K_{I D_{S}}\right)=\hat{e}\left(H\left(I D_{S}\right), S K_{I D_{D}}\right.$ in this way, it can prove $D$ is the destination node. The destination D maintains the route table (Fig. 6).

\begin{tabular}{|c|l|l|c|r|}
\hline sequence & Dest_ID & Ancestor & Successor & TTL \\
\hline Seq\# & $\mathrm{H}_{1}\left(\mathrm{ID}_{\mathrm{S}}\right)$ & In_addr & N/A & $\mathrm{T}_{\mathrm{S}}$ \\
\hline \multicolumn{6}{|c}{ Fig. 6. Destination node changes its route table. }
\end{tabular}

\section{2) Inter-group anonymous route request}

When a node want to communicate with the node in the different groups, the source node $S$ will also generate the relevant parameters and add the network identity $N F$ into the ARREQ. The following form as follows: $A R R E Q=<R R E Q$, seq\#, NF, HopCount, Cs $>$

Intermediate nodes will discard the ARREQ packet as long as find $N F$, which means the destination node is in the other group. After that, change their route tables like intra communication.

When the cluster head node receives the ARREQ, it will know the source node want to communicate with the other node of the other subgroups. Because the system uses a multi-band classification, cluster head node use another frequency to forwards the ARREQ to its neighbor cluster head nodes. After that, it changes its route table.

When the neighbor cluster head node receives the RREQ, it checks $N F$, if not, forward, otherwise, discard the $N F$ of ARREQ. ARREQ $=<R R E Q$, seq\#, HopCount, Cs $>$.

In this way, it can find the destination node by intra routing discovery. Intermediate nodes complete their route tables. Finally, it can achieve the inter-group routing node discovery.

\section{E. Route Reverse Phase}

Inter-group route and intra-group route reverse both executed as follows. The nodes receive the packet from the neighbor honest nodes and can find its forward nodes by seq\#.

Step 1 . D randomly select $b \in[1, p-1]$, computers $g^{b}$ and $H\left(g^{b}\left\|K_{S D}\right\| 1\right)$, where $M_{D}=\left[H_{1}\left(I D_{S}\right), I D_{D}, g^{b}, H\left(g^{b}\left\|K_{S D}\right\| 1\right)\right]$ $D$ use $P K_{S}=H_{1}\left(I D_{N}\right)$ to encrypt $M_{D}$, as $C_{D}=E_{P K_{S}}\left(M_{D}\right)$.
According to seq\# look up its upstream, and appends authentication tag encrypt message $C_{D}$ together with seq\# using secret key $K_{D I_{n}}$ (key exchange based on the ring signature) shared with the upstream. $D$ sends ARREP to $I_{n}$, which is formatted as follows: $A R R E P=<R R E P$, seq\#, $C_{D}, M A C_{K_{D I_{n}}}\left(r t_{-}\right.$seqno, $\left.C_{D}\right)>$ computes shared session key $S K_{S D}=\left(g^{a}\right)^{b}$.

Step 2. $D$ uses its shared secret key $K_{D I_{n}}$ to verify $M A C_{K_{D I_{n}}}\left(r t_{-}\right.$seqno, $\left.C_{D}\right)$, If seq\# is found in its local table, it continues, otherwise, it drops. If authentication fails, it drops the ARREP, otherwise, it continues.

Step 3. When the source node $S$ receives ARREP, it can authenticate, $S$ also can compute session key $S K_{S D}=g^{a b}$, the data packet transmission start the source to destination node is established.

\section{F. Data Forwarding Phase}

Step 1. $S$ uses session key to encrypt $M$ as $C=E_{S K_{S D}}(M)$, it can find its downstream node $I_{1}$ from its local route table, and uses their session key to encrypt $\mathrm{C}$, seq\# as $M A C_{K_{S I_{1}}}(C)$, $R_{I_{1}}=e K_{S I_{1}}(\mathrm{seq} \#)$. At last, it sends $\left(R_{I_{1}}, C, M A C_{K_{S I_{1}}}(C)\right)$ to node $I_{1}$.

Step 2. When $k_{a b}=x(y P) \cdots$ Alice receiving the packet, $=y(x P) \cdots B o b$

node use its public key to authentication. If success, decrypt $R_{I_{1}}$, and then lookup downstream by seq\# from local route table. At last, it changes the information of packet by their session key.

Step 3. When the destination receives $\left(R_{D}, C, M A C_{K I_{N} D}(C)\right)$, it uses its shared secret key to verify $M A C_{K I_{N} D}(C)$. If it success, decrypting $R_{D}$. It finds the corresponding session key $S K_{S D}=g^{a b}$, which is taken to recover $M$. Similarly $D$ also can send confidential data to $S$ in the same way.

\section{Protocol Analysis Based on UC Model}

The SI unit for magnetic field strength $H$ is $\mathrm{A} / \mathrm{m}$. However, if you wish to use units of $T$, either refer to magnetic flux density $B$ or magnetic field strength symbolized as $\mu_{0} H$. Use the center dot to separate compound units, e.g., "A· $\mathrm{m}^{2}$."

The proposed protocol $\pi$ can be divided into three sub-agreement $\pi_{1}, \pi_{2}, \pi_{3} . \pi_{1}$ is the authentication key exchange phase; $\pi_{2}$ is anonymous routing stage; $\pi_{3}$ is the data transfer phase. According to common characteristics, the security of $\pi$ is equivalent $\pi_{1}, \pi_{2}, \pi_{3}$ combination of security.

Lemma 1: The protocol $\pi_{1}$ meet the UC security is based on the ring signature, which can against adaptively chosen message attack.

Proof: [5] reference.

Lemma 2: The protocol $\pi_{3}$ meet the UC security. 
Proof: On the basis of the protocol $\pi_{1}$, neighborhood nodes get their MAC session key. Source node and destination node establish session key by select random number $a, b$ and sharing parameters $g$, which meet the bilinear difficulties in problem solving. So the data transfer is the UC security [8].

Definition 1: the ideal route request process $F_{A R R E Q}$ [9], $\mathrm{F}_{\mathrm{ARREQ}}$ execute instruction under the background of the security parameter $k$, participates $P_{1}, \ldots, P_{n}$, and adversary $\mathrm{S}$.

$\mathrm{F}_{\text {ARREQ }}$ internal structure: the path of $P_{i}$ is $B_{i}$. The record entry form $\left(\operatorname{sid}, P_{s}, P_{j 1}, P_{j 2}, \ldots, P_{i}\right)$, where $P_{s}$ is the source node, $P_{j 1}, P_{j 2}$ are intermediate nodes. Bad represent those compromised nodes. Ead means those eavesdropping nodes. Text symbol " $\rightarrow$ " indicates that the message flow from left to right; " $\wedge$ " is the logical and; " $\vee$ " is logical or; $N B_{A}\left(P_{i}\right)$ is the $P_{i}$ 's neighbor set; $N B_{H}\left(P_{i}\right)$ is the $P_{i}$ 's honest set of neighbors. The communication channel is $C_{I}$.

Lemma 3: protocol $\pi_{2}$ is UC anonymous in the $F_{S C}$ [6] hybrid model.

Proof: Set the attack $A$ running in the routing request of the $F_{S C}$ model. We construct an attack $S$ of $F_{A R R E Q .} S$ runs a simulated $A$. The purpose of simulation is to prove that the environment $Z$ can't distinguish it interacts with $S$ of $F_{A R R E Q}$ or interacts with $A$ in the $\mathrm{F}_{\mathrm{SC}}$ hybrid model. $S$ simulates $F_{S C}$.

$S$ maintains two tables: parameter table list, which is used to send ARREQ to A; message list List $_{A R R E Q}$ which is used to send message to $F_{A R R E Q}$. When $S$ receives $N B_{A}\left(P_{i}\right)$ ARREQ for the first time, it randomly generated seq\#, one-time key pair $\left(E_{p k}, E_{s k}\right)$, and use the destination public key to encryption $M_{s}$, saving ( sid, seq\#, $E_{p k_{D}}\left(M_{s}\right)$ ) to the list. $S$ eavesdrops $P_{x}$. when it received $\left(N B_{A}\left(P_{i}\right), A R R E Q\right)$ from $C_{I}$, looking up List ( sid, seq\#, $E_{p k_{D}}\left(M_{s}\right)$ ) by sid. It will pack the message into $m$ of ARREQ. $S:\left(N_{i}, N_{x}, m\right) \rightarrow A, S$ simulate $N_{X}$ (corresponding to $P_{x}$ of $F_{A R R E Q}$ ), receive $A R R E Q$ from $N_{i}$ forwarded. That means $\mathrm{S}$ simulates $N_{X}$ to receive and forward the packet of ARREQ.

If $P_{x} \in B a d$ received $\left(N B_{A}\left(P_{i}\right), A R R E Q\right)$, then $S:\left(N_{i}, N_{x}, m\right) \rightarrow A$, where the message $m$ is the same with the contents of eavesdropped. Then, it uses private key of $P_{X}$ to decrypt $E_{p k_{D}}\left(M_{s}\right)$ of message $m$ and send the result to $A$. Save $\left(N_{i}, N_{x}, m\right)$ to list $_{A R R E Q}$. Assume the nodes in the path from $N_{x}$ to $N_{y}$ were captured, $N_{y}$ forward the rout request $m$ to $N_{j}\left(N_{j} \notin \mathrm{Bad}\right)$ from $A$ to $S$. $S$ get $\left(\right.$ sid $\left., N_{i}, N_{x}, m\right)$ $S:\left(P_{i}, P_{y 1}^{\prime}, P^{\prime}{ }_{y 2}, \ldots, P_{y}^{\prime} P_{j}, o k\right) \rightarrow F_{A R R E Q}$ by looking up the recorded List $_{A R R E Q}$, if $A R R E Q$ and list $_{A R R E Q}$ have no corresponding date, discarded the packet.

We define the sequence of mixing machine environment proves that process simulation. The probability that $Z$ is able to distinguish between an interaction with $A$ and real parties running protocol $\pi_{2}$ and an interaction with $S$ and dummy parties accessing $F_{A R R E Q}$ is at most a negligible probability.

Hybrid $H_{y 0}$. It generates key pairs for all honest nodes and execute the protocol $\pi_{2}$ for each node. According the protocol $\pi_{2}$ it can simulate the host nodes interact with $A$ and $Z$. The interaction output of $Z$ and $H_{y 0}$ is the same with the output of $Z$ in the real-life world. Hybrid $H_{y 1}$ and $H_{y 0}$ is basically the same, the differences is that: if $P_{x} \in E a d, N_{x}$ forwards the packet $A R R E Q$ to $N_{i}, H_{y 1}$ gets the route path by decryption ARREQ. If the route was not recorded before, then this path is marked as false path Hybrid $H_{y 2}$ and $H_{y 1}$ is basically the same, the differences is that: upon $P_{x} \in N B_{A}\left(P_{i}\right) \wedge\left(P_{x} \in \operatorname{Bad} \vee\right.$ $\left.P_{x} \in \mathrm{Ead}\right) \wedge P_{i} \notin \mathrm{Bad}$, when $N_{i}$ forwards the routing request to $N_{x}, H_{y 2}$ uses private key to decrypt the message of $E_{p k_{D}}\left(M_{s}\right)$, and sends the result to attack $A$. Hybrid $H_{y 3}$ and $H_{y 2}$ is basically the same, the differences is that: if $P_{i} \notin \mathrm{Bad}$ $\wedge P_{x} \in N B_{A}\left(P_{i}\right) \wedge\left(P_{x} \in B a d \vee P_{x} \in\right.$ Ead $)$ when $N_{i}$ forward the routing request to $N_{x}, H_{y 3}$ sends the route request to attack $A$. Hybrid $H_{y 4}$ and $H_{y 3}$ is basically the same, the differences is that: $H_{y 4}$ do not execute protocol $\pi_{2}$ for honest nodes, but get the path through the ideal functionality, that means it don't execute any encryption or decryption. The output of $Z$ and $H_{y 4}$ interaction is the same with the output of $Z$ in $F_{A R R E Q}$. If $H_{y i}$ and $H_{y i+1}(i=0,1,2,3)$ is interacting, the output of $Z$ is the same, the protocol $\pi_{2}$ is anonymous. The signature of against Chosen-message attacks is to guarantee the the success probability of tampering honest node path can be ignored.

The output of $Z$ and $H_{y 1}, H_{y 2}$ interaction can't computationally indistinguishable. If it can distinguish, consider another sequence of mixing machine $H_{y l}$, before the condition of $H_{y 2}$ is first time satisfied, $H_{y l}$ did what $H_{y l}$ should do. Then execute according to $H_{y 2}$. In the polynomial sector, $H_{y 1}$ and $H_{y 1}$ will be undistinguishable. Otherwise, the adversary can declassification the IND-CPA security of public key encryption. Assume challenge nodes $N$, an optional node $N_{j}$ let $\left(N_{j}, S K_{j}\right)=\left(N, S K_{N}\right)$; challenger generates key-pairs for the other nodes. When execute the mix machine for $i+1$ times, let challenger public key is $E_{P K}$, if it can distinguish that means to crack the security of IND-CPA.

The output of $Z$ and $H_{y 2}, H_{y 3}$ interaction is computationally indistinguishable. UC secure channel proves the indistinguishable of the ciphertext, which is generated by different plaintext.

The output of $Z$ and $H_{y 3}, H_{y 4}$ interaction is computationally indistinguishable. The reason is the ideal functionality executes the function of honest nodes in the $\mathrm{Hy}_{3}$.

Theorem 1 anonymous routing protocols $\pi$ can achieve UC security Proof. It is proved by Lemma 1-Lemma 3.

\section{COMPARISON WITH RELATED WORK}

TABLE I: COMPARISON OF THE PROPOSED SCHEME AND RELATED WORK

\begin{tabular}{|l|c|c|c|c|}
\hline & $\begin{array}{c}\text { ANODR } \\
{[10]}\end{array}$ & $\begin{array}{c}\text { MASK } \\
{[11]}\end{array}$ & $\begin{array}{c}\text { ASRPAK } \\
\text { E[3] }\end{array}$ & OURS \\
\hline $\begin{array}{l}\text { Sender } \\
\text { anonymity }\end{array}$ & $\times$ & $\times$ & $\times$ & $\sqrt{ }$ \\
\hline $\begin{array}{l}\text { anonymous } \\
\text { authentication }\end{array}$ & $\times$ & $\times$ & $\sqrt{ }$ & $\sqrt{ }$ \\
\hline $\begin{array}{l}\text { Anonymous } \\
\text { routing }\end{array}$ & $\times$ & $\times$ & $\sqrt{ }$ & $\sqrt{ }$ \\
\hline $\begin{array}{l}\text { hierarchical } \\
\text { routing }\end{array}$ & $\times$ & $\times$ & $\times$ & $\sqrt{ }$ \\
\hline UC security & $\times$ & $\times$ & $\times$ & $\sqrt{ }$ \\
\hline
\end{tabular}


Our protocols can provide end-to-end anonymity of a route, sender anonymity, the security of authenticated session key shared. In addition, the protocols suitable for hierarchical ad hoc networks and achieve UC security (Table I).

\section{CONCLUSION}

In this paper, we proposed a new anonymous routing based on the anonymous ring signature authentication routing protocol, the protocol is more suitable for large-scale ad hoc network and achieve the sender anonymity, routing anonymity. At last, we use the UC model to prove the security of this protocol. As the future research, we plan to improve route efficiency.

\section{REFERENCES}

[1] L. chaumd, "Untraceable electronic mail, return dresses, and digital pseudonyms," Communications of the ACM, vol. 24, no. 2, pp. 84-88, 1981.

[2] S. S. Varghese and J. I. J. Raja, "A survey on anonymous routing protocols in MANE," in Proc. the 12th International Conference on Networking, 2010.

[3] ASRPAKE, "An anonymous secure routing protocol with authenticated key exchange for wireless Ad hoc networks," in Proc. the ICC 2007.

[4] A. Bender, J. Katz, and R. Morselli, "Ring signatures: Stronger definitions and constructions without random oracles," in TCC 2006 , pp. 60-79.
[5] K. Yoneyama and K. Ohta, "Ring signatures," IPSJ Digital Courier, vol. 3, pp. 571-584, 2007.

[6] R. Canetti. Universally composable security. [Online]. Available: http://eprint.iacr.org/2000/067.

[7] W. D. Yang, "Weight-based clustering algorithm for mobile ad hoc network," in Proc. Cross Strait Quad-Regional Radio Science and Wireless Technology Conference, 2011, pp. 787-791.

[8] J. Camenisch and A. Lysyanskaya, A Formal Treatment of Onion Routing, Berlin: Springer, 2005.

[9] Z. Yang, "Formal treatment of an anonymous on demand routing protocol in MANET," Journal of Computer Research and Development, 2008.

[10] K. Kong and X. Hong, "ANODR: an anonymous on demand routing with untraceable routes for mobile ad hoc networks," in Proc. MobiHoc'03, 2003, pp. 291-302.

[11] Y. Zhang, W. Liu, and W. Lou, "Anonymous communications in mobile ad hoc networks," in Proc. the 24th Annual Joint Conference. IEEE, 2005, pp. 1940-1951.

Yuan Xiaopeng was born in 1981. He recevied a bachelor degree. He is now working as an engineer in High-Level School Admissions Office of Gansu Province, Lanzhou, China. His research areas are in software design and information security.

Feng Tao was born in 1970, he recevied a $\mathrm{PhD}$ degree and has been a supervisor of School of Computer and Communication Lanzhou University of Technology, Lanzhou, China. His research areas are in networking and information security. 